\title{
Phytochemicals of lentil (Lens culinaris) and their antioxidant and anti-inflammatory effects
}

\author{
Bing Zhang ${ }^{\mathrm{a}}$, Han Peng ${ }^{\mathrm{a}}$, Zeyuan Deng ${ }^{\mathrm{a}}$ and Rong Tsao $\mathrm{b}^{\mathrm{b}^{*}}$
}

\begin{abstract}
a State Key Laboratory of Food Science and Technology, Nanchang University, Nanchang, 330047, China
${ }^{\mathrm{b}}$ Guelph Research \& Development Centre, Agriculture and Agri-Food Canada, 93 Stone Road West, Guelph, ON N1G 5C9, Canada

*Corresponding author: Rong Tsao, Guelph Research \& Development Centre, Agriculture and Agri-Food Canada, 93 Stone Road West, Guelph, ON N1G 5C9, Canada. Tel: +1 226-217-8108; Fax: +1 226-217-8183; E-mail: rong.cao@agr.gc.ca

DOI: $10.31665 / \mathrm{JFB} .2018 .1128$
\end{abstract}

Received: January 18, 2018; Revised received \& accepted: February 26, 2018

Citation: Zhang, B., Peng, H., Deng, Z., and Tsao, R. (2018). Phytochemicals of lentil (Lens culinaris) and their antioxidant and antiinflammatory effects. J. Food Bioact. 1: 93-103.

\begin{abstract}
Lentils contain a plethora of bioactive phytochemicals such as extractable and insoluble-bound phenolics, carotenoids, tocopherols, saponins, phytic acid, and phytosterols, which have been increasingly attributed to the health benefits of lentil consumption in the diet. The concentration and stability of these phytochemicals in lentils may be affected by several processing parameters including different thermal processing, exogenous enzyme treatment and germination. Consumption of lentils has been associated with the risk reduction of many diseases due to the potential antioxidant activity and anti-inflammatory potential of phytochemicals in lentils. This mini review is intended to provide most current information on the phytochemical composition of lentils, and the potential antioxidant and anti-inflammatory properties of these compounds.
\end{abstract}

Keywords: Lentils; Phytochemicals; Phenolics; Carotenoids; Antioxidants; Anti-inflammatory.

\section{Introduction}

Legumes, commonly referred to as "pulses", are one of the most extensively cultivated crops throughout the world and are typically marketed as dry products (Schneider, 2002). They are consumed as a basic staple food in many countries, providing ideal protein, carbohydrates (including dietary fibers), fatty acids, minerals, and vitamins complementary to cereal-based diets (Gumienna et al., 2009; Marathe et al., 2011). Pulses are defined by the Food and Agriculture Organization (FAO) of the United Nations as grain legumes harvested only for their seeds (Mudryj et al., 2012). FAO lists eleven primary pulses including lentils, and excludes leguminous seeds used primarily for oil extraction, such as soybeans and groundnuts, or those consumed in immature form as vegetables, such as green beans and green peas (Dahl et al., 2012). Lentils (Lens culinaris) have been gaining increasing attention for their nutritive value as human diet. It is sometimes called "poor man's meat", which originated in ancient Europe (Bhatty, 1988). For these reasons, lentils have long been recognised as an inexpensive, excellent alternative to animal proteins, and are considered as a potential whole food source for people affected by micronutrient malnutrition (Thavarajah et al., 2009). Lentil crop was first introduced into southern Manitoba and Saskatchewan in Canada during the grain surplus years of the early 1970's due to favorable price compared with depressed cereal prices (Bhatty, 1988). Canada is by far the world's largest lentil exporter to the global marketplace, selling to over 100 countries each year, and produces about $25 \%$ of the total world output (Thavarajah et al., 2009). The most commonly grown lentil cultivars are the large green "Laird" cultivar and the red lentil (http://www.pulsecanada.com/food-health/whatis-a-pulse/lentil).

In recent years, many studies have shown potential health benefits of pulses, including lentils, beyond satisfying basic nutrient requirements for humans (Rochfort and Panozzo, 2007). Epidemiological and interventional studies suggest that pulse consumption is inversely associated with the incidence of several chronic diseases, such as coronary heart disease, type II diabetes mellitus, cardiovascular diseases, cancer and aging (Amarowicz and Pegg, 
2008; Villegas et al., 2008). Some of these purported benefits of consuming a pulse-based diet have been attributed to their high content of phytochemicals that exert antioxidant and anti-inflammatory activity in vitro and in vivo (Amarowicz et al., 2004; Xu and Chang, 2012). However, the consumption of pulses like lentils is limited in western countries, with only about one in eight people consuming pulses on a daily basis (Mudryj et al., 2012), due to traditional eating customs, lack of consumer education and understanding on the nutritional values, processing techniques and available diversified food products. Despite this, incorporation of pulses into western diets has been highly recommended for consumers to receive maximum health benefits (Aguilera et al., 2010). In order to heighten public awareness of the nutritional benefits of pulses as part of sustainable food production aimed towards food security and nutrition, FAO has declared 2016 the International Year of Pulses (IYP) (http://www.fao.org/pulses-2016/en/).

Macronutrients such as carbohydrates and proteins are the main components of lentils, and current research suggests protein hydrolysates and peptides may be responsible for some of the observed health benefits, and dietary fibres and their colonic fermentation products i.e. short chain fatty acids (SCFA) may be a contributing factor to gut and colon health. Micronutrients such as phenolics have also shown strong antioxidant and anti-inflammatory effects. However, despite these findings, there is no consensus on the exact bioactive component(s) in lentils that contribute to the health benefits. There is a need for a comprehensive overview on the possible bioactive components in lentils. Inflammation, especially low grade inflammation, is the root cuase of oxidative stress induced chronic diseases. Food components with antioxidant and anti-inflammatory activities therefore are naturally targets of the health benefits of lentils. The present contribution intends to provide a review of the recent advances in the phytochemicals of lentils and their antioxidant and anti-inflammatory effects and how they might contribute to reduction in human health risks.

\section{Phytochemicals in lentils}

In general, phytochemicals could be broadly defined as all plant derived chemicals including macronutrients such as carbohydrates, lipids and proteins. However, in the present contribution, the term "phytochemicals" refers to those small non-essential bioactive compounds or secondary metabolites that occur naturally in plants. These phytochemicals such as flavonoids and carotenoids are usually responsible for the color and other organoleptic properties of food, but also have other significant biological activities. Lentils have been reported to contain an array of different phytochemicals such as phenolics, condensed tannins, carotenoids, tocopherols, saponins, phytic acid, and phytosterols, making them the major sources for phytochemicals in the diet. Table 1 summarizes the different phytochemicals reported for lentils.

\subsection{Extractable phenolics}

Polyphenolic compounds are perhaps the most diverse group of phytochemicals that are known for their various biological properties. To date, more than 8,000 polyphenolic substances have been identified. These polyphenols can be classified into sub-groups such as phenolic acids and flavonoids according to their molecular structures. Phenolic acids are simple phenolics that can be further divided into hydroxybenzoic acid derivatives and hydroxycinnamic acid derivatives. Flavonoids are the most important phe- nolic subclass, characterized by a $\mathrm{C}_{6}-\mathrm{C}_{3}-\mathrm{C}_{6}$ backbone structure. Flavonoids can be further classified into different sub-groups of flavan-3-ols, flavonols, flavones, flavanones, anthocyanidins and isoflavones, and oligomers such as proanthocyanidins. The phenolic compounds in plant are conventionally extracted by ethyl or methyl alcohols, acetone or their aqueous mixtures, which is only efficient in extracting soluable phenolics., leaving behind other phenolics that exist in the bound form. For this reason, the majority of the reported measurement of phenolics in food samples is confined to the soluble or extractable phenolic fraction.

Lentils are a significant dietary source of these extractable phenolics. The major phenolic compounds found in lentils include sub-classes of phenolic acids, flavan-3-ols, condensed tannins (proanthocyanidins), anthocyanidins, flavonols, stilbenes, flavones, and flavanones. Lentil was reported to have the highest total phenolic content (TPC) of $7.53 \mathrm{mg}$ gallic acid equivalents (GAE)/g dry weight (DW) among 8 different types and varieties of pulses, as well as $2.21 \mathrm{mg}$ catechin equivalents $(\mathrm{CE}) / \mathrm{g}$ DW of total flavonoids content (TFC) (BJ Xu \& Chang, 2007). Lentils also contain 1.5-2.6 mg/g of total phenolic acids (Xu and Chang, 2010). Amarowicz et al. $(2009,2010)$ identified 24 phenolic compounds including phenolic acids and flavonoids in the extractable fraction of red lentil and 20 in green lentil. They found that $p$-hydroxybenzoic acid, trans- $p$-coumaric acid, trans-ferulic acid and sinapic acid were major phenolic acids in red lentil, whereas trans- $p$-coumaric acid and trans-ferulic acid were mainly present in green lentil. The content of individual flavonoids in lentils ranged from 0.27 to 289 $\mu \mathrm{g} / \mathrm{g}$ DW. Flavonols, flavan-3-ols and condensed tannins (proanthocyanidins), such as kaempeferol glycoside, quercetin diglycoside, catechin, epicatechin, prodelphinidin dimer and digallate procyanidin, were identified as the predominant flavonoids of lentils in the study reported by Zou et al. (2011). Alshikh et al. (2015) further fractionated the crude phenolic extract of lentils and found that majority of the extractable phenolics were in esterified form (2.32-21.54 mg GAE/g) rather than in free form (1.37-5.53 mg $\mathrm{GAE} / \mathrm{g})$. Catechin, epicatechin and procyanidins B were predominant flavonoids in both free and esterified fractions of all tested lentils. They also identified and quantified methyl vanillate and prodelphinidin dimer $\mathrm{A}$ in lentils for the first time. Others have found that monomeric flavan-3-ols (catechin and epictechin), as well as limited amount of flavone (luteolin) were present in 11 selected lentil cultivars (Xu and Chang, 2010). Our recent study revealed that flavonols such as kaempeferol glycoside, and flavan-3-ols mainly catechin and epicatechin glycosides were the predominant phenolics in the aqueous methanolic extract of 20 lentil cultivars, and it was these compounds that contributed most to the antioxidant capacity of lentils (Zhang et al., 2015). The varied flavonoid compositions in lentils among published data might be attributed to the different genotype, growing environment, extraction condition and chromatography system. Anthocyanins are pigmented flavonoids found in dark colored lentil varieties, and there is very limited information available in the literature. Early studies showed that lentil seeds contained a compound that resembled a diglycosyl derivative of delphinidin (D’Arcy and Jay, 1978). Takeoka et al. (2005) first isolated a major anthocyanin from the acidified methanolic extract of Beluga black lentils, and identified as delphinidin 3-O-(2-O- $\beta-D-$ glucopyranosyl- $\alpha$-L-arabinopyranoside).

The distribution of phenolic compounds differs greatly in the cotyledon and the seed coat of lentils. Although the lentil seed coat accounts for only 8 to $11 \%$ percent of the whole seed weight, it provides significant contribution to the overall benefits of lentils (Duenas et al., 2006). Mirali et al. (2014) reported that phenolic compounds in lentil seed coat were more abundant and diverse than in the cotyledon. The seed coat was found to contain a large amount 


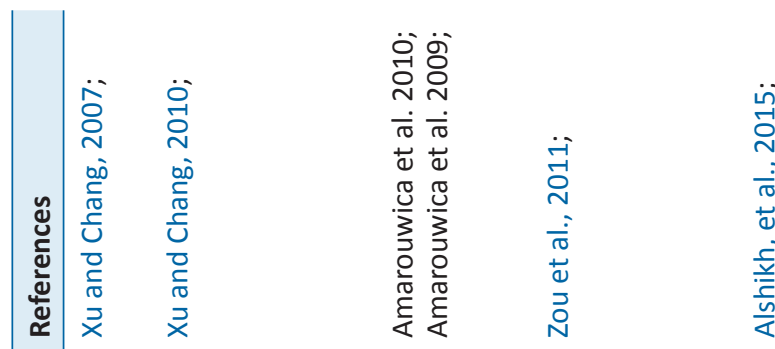

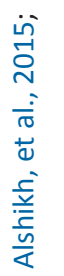

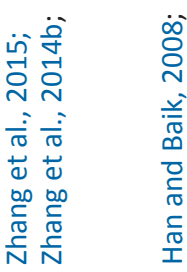

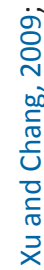
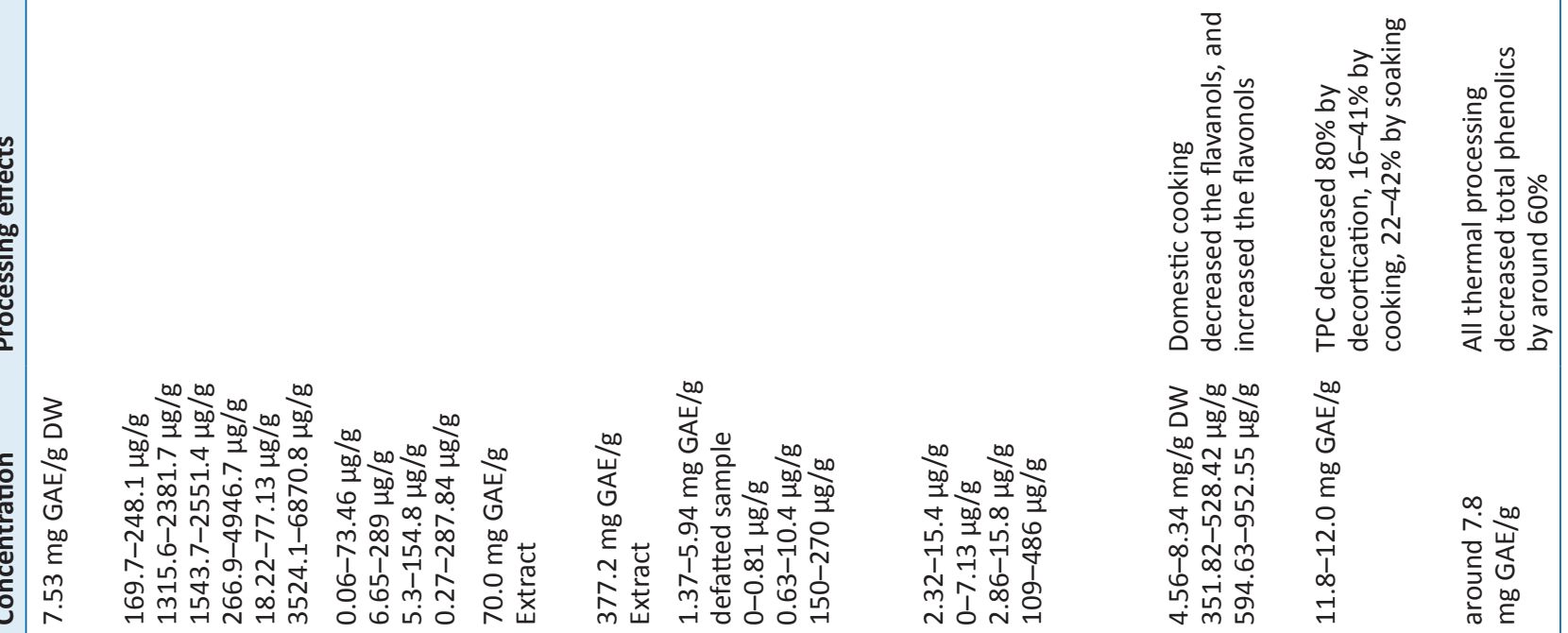

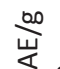

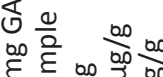

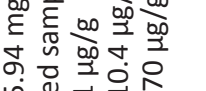

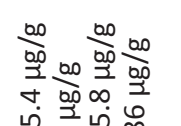

$\sum_{0}^{\infty} \frac{\infty}{20}$

崖

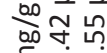

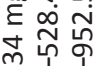

$\infty \stackrel{1}{\sim} m$

b

국ำ

mîn

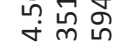

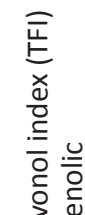

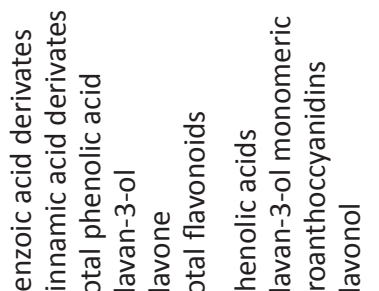

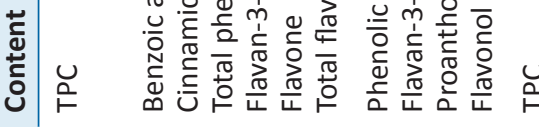

논
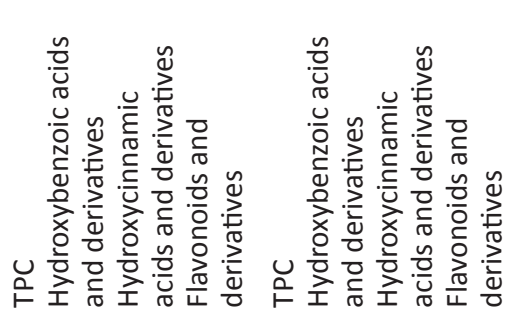

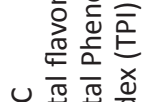

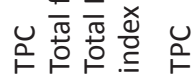

얀

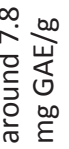

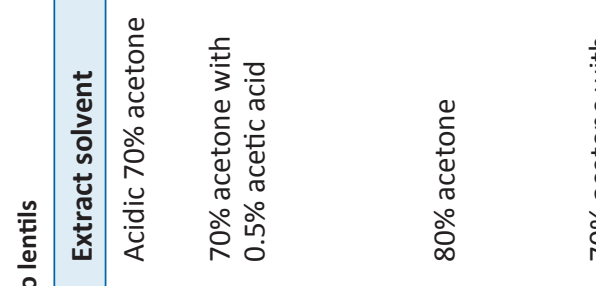

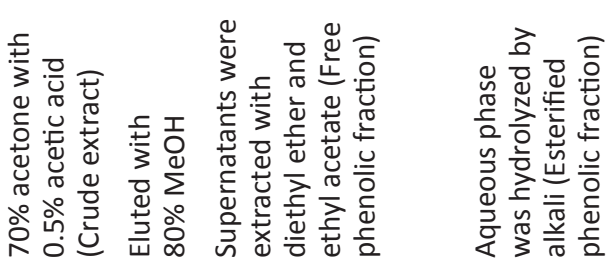

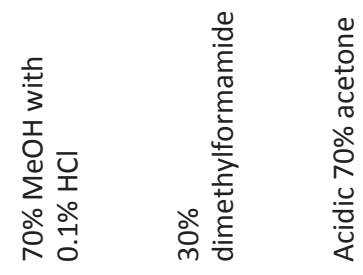

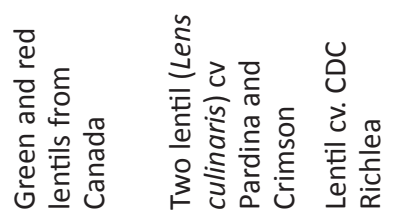




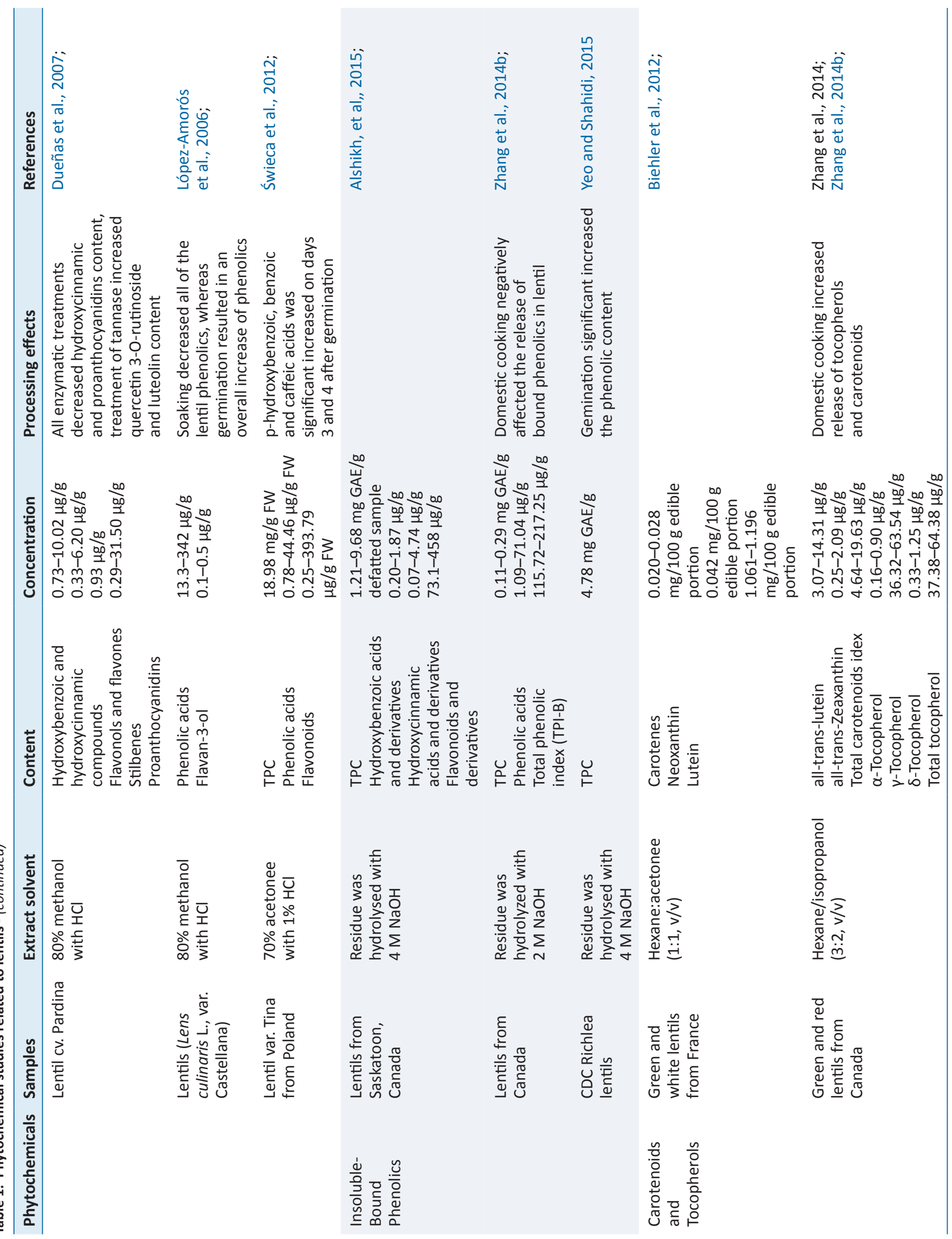




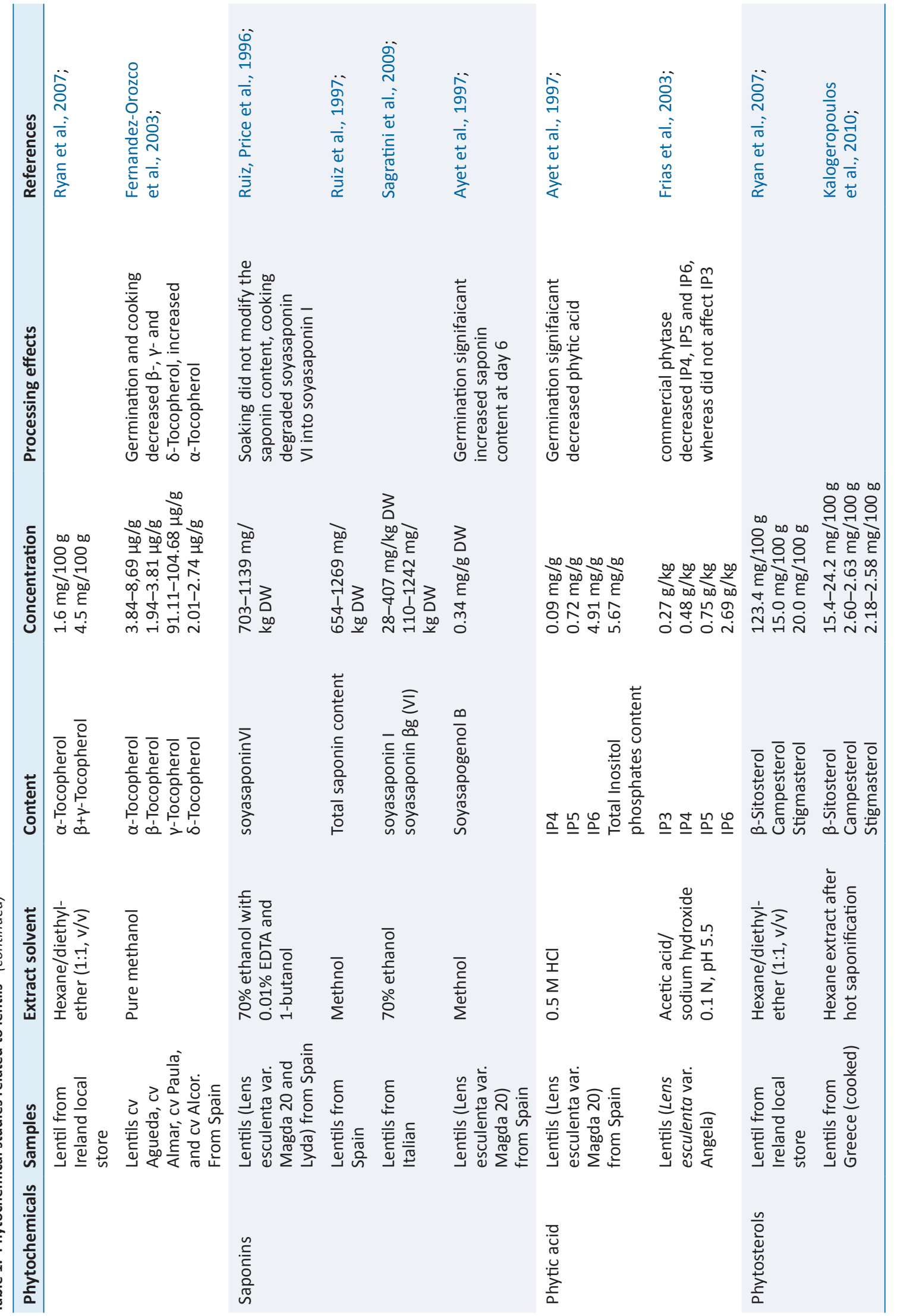


of monomeric flavan-3-ols, proanthocyanidin oligomers and polymers, as well as small amounts of glycosides of flavonols such as quercetin, myricetin, luteolin, and apigenin. Phenolic acids, on the other hand, such as hydroxybenzoic and hydroxycinnamic acids, in both free and bound forms, were mainly present in the cotyledon of lentils (Dueñas et al., 2002). Interestingly, a stilbene trans-resveratrol-5-glucoside was identified for the first time in the seed coat of lentils (Dueñas et al., 2002). The same research group further reported the structural compositions of proanthocyanidins, the major group of polyphenols present in the seed coat of lentils (Dueñas et al., 2003). The proanthocyanidins in the seed coat of lentil mainly included monomeric, oligomeric, and polymeric flavan-3-ols. The major monomeric flavan-3-ol was (+)-catechin-3-glucose, with lesser amounts of $(+)$-catechin and (-)-epicatechin aglycones. Various dimer, trimer, and tetramers constituted of catechin, gallocatechin, and catechin gallate units were identified in the oligomeric fraction, and several procyanidins and prodelphinidins from pentamers to nonamers in the polymeric fraction. Approximately $65-75 \%$ of proanthocyanins in the seed coat of lentils are polymers with 7-9 $\mathrm{mDP}$ (mean degree of polymerization), and $20-30 \%$ are oligomers with an $\mathrm{mDP}$ of $4-5$. Proanthocyanins are strong antioxidants and have been reported to have many health beneficial effects, thus it is highly recommended that lentils are consumed in-whole.

\subsection{Insoluble/Bound phenolics}

Apart from most studied extractable phenolics, there still exist easily neglected insoluble or bound phenolics that are commonly associated with cell wall materials. The lack of assessing bound phenolics leads to underestimation of the total phenolic content and the overall actual health benefits of lentils or other pulses. In general, organic solvents such as ethanol, methanol and acetonee are employed to directly extract the soluble phenolics (extractable phenolics) in plants, whereas insoluble-bound phenolics can only be released upon alkali, acid, or enzymatic treatment of samples before extraction (Andreasen et al., 2001; Bartolome and Gómez-Cordovés, 1999; Zupfer et al., 1998). The insoluble-bound phenolics are considered to contribute additional health benefits because they may avoid degradation under upper gastrointestinal digestion conditions, and are absorbed into teh circulation system or epithelial cells after being released by intestinal microflora fermentation (Andreasen et al., 2001). However, unlike other plants such as cereals, fruits and vegetables, insolublebound phenolics of pulses, particularly lentils, and their potential contribution to health benefits have not been well studied. Han and Baik (2008) found that bound phytochemicals contributed more to total antioxidant activity in lentils than free (extractable) phytochemicals. The TPC of insoluble-bound phenolics in lentils ranged from 0.18 to $17.5 \mathrm{mg} \mathrm{GAE} / \mathrm{g}$, whereas TFC ranged from 0.03 to $4.13 \mathrm{mg} \mathrm{CE} / \mathrm{g}$ (Alshikh et al., 2015; Zhang et al., 2014b). Alshikh et al. (2015) identified 15 compounds including hydroxybenzoic acids, hydroxycinnamic acids, flavonoids and their derivatives in the insoluble-bound fraction of lentils. Catechin, catechin-3-glucoside and procyanidin dimer Bweree the predominant phenolic compounds identified in the insoluble-bound fractions of 6 tested lentil cultivars. In our previous study, five phenolic compounds including gallic acid, protocatechuic acid, catechin, epicatechin and 3-hydroxycinnamic acid were identified in the insoluble-bound fractions of lentils (Zhang et al., 2014b).

\subsection{Carotenoids and tocopherols}

Carotenoids and tocopherols are well-known lipophilic antioxi- dants that are synthesized in whole or in part from the plastid isoprenoids (DellaPenna and Pogson, 2006). Carotenoids comprise a large isoprenoid family and most are C40 tetra-terpenoids derived from phytoene. It provides plants with distinctive red, orange, and yellow colours. Tocopherols are a group of nutrients that constitutes vitamin $\mathrm{E}$ that are essential to health of all mammals. Lentils are a good source of both carotenoids and tocopherols. Biehler et al. (2012) reported presence of lutein, carotenes and neoxanthin in lentils, among which lutein (1.061-1.196 mg/100 g edible portion) was the major carotenoid. Our recent study examined the carotenoid and tocopherol compositions in 20 lentil cultivars (10 green and 10 red) (Zhang et al., 2014a). All-trans-lutein accounted for $64-78 \%$ of the total carotenoid content (TCC) in lentils, followed by all-trans-zeaxanthin, which constitutes $5-13 \%$ of TCC. The total lutein and zeaxanthin contents (total of all-trans and cisisomers) in lentils ranged from $4.32-17.29 \mu \mathrm{g} / \mathrm{g}$ DW to $0.32-2.73$ $\mu \mathrm{g} / \mathrm{g}$ DW, respectively. Total tocopherols of lentils were from 37 $64 \mu \mathrm{g} / \mathrm{g} \mathrm{DW}$, predominantly $\gamma$-tocopherol (96-98\% of the tocopherol content), followed by $\delta$ - and $\alpha$-tocopherols. Similar results were repored by Ryan et al. (2007) who did not separate all tocopherol isomers. All $\alpha-, \beta$-, $\gamma$ - and $\delta$-tocopherol isomers were reported in lentils in another study (Fernandez-Orozco et al., 2003). When consumed daily, these carotenoids and tocopherols of lentils can provide substantial nutritional and health benefits.

\subsection{Saponins}

Saponins are a diverse group of compounds characterized by a carbohydrate moiety attached to a steroid or triterpenoid aglycone in their structures. These phytochemicals have long been considered undesirable due to their toxicity and haemolytic activity. Saponins in pulses such as lentils however are attracting considerable interest in recent years due to their ability to lower plasma cholesterol levels in human and suppress cancer growth. Pulses including lentils are considered among the best sources of saponins. Total saponin content of lentils has been reported to be in the range of $654-1269 \mathrm{mg} / \mathrm{kg} \mathrm{DW}$, and the main saponins were soyasaponin I and VI in Spanish lentils (Ruiz et al., 1996; Ruiz et al., 1997). Sagratini et al. (2009) tested 32 Italian lentils, and found soyasaponin $\mathrm{I}$ and soyasaponin $\beta \mathrm{g}$ (also known as VI) to be $28-407 \mathrm{mg} / \mathrm{kg}$ and $110-1242 \mathrm{mg} / \mathrm{kg}$.

\subsection{Phytic acid}

Phytic acid (or inositol hexaphosphate, IP6) is a saturated cyclic acid, and is considered as the major source of phosphorus in many plant tissues or organs, especially in seeds. The catabolites of phytic acid are called lower inositol polyphosphates including inositol tri- (IP3) , tetra- (IP4) and penta-phosphate (IP5). Phytic acid has been proposed to serve a vital role in protecting the seeds against the deleterious effects of oxygen and iron (Graf and Eaton, 1990). Lentils have been reported to contain $86 \%$ of IP6 (4.91 mg/g), $13 \%$ of IP5 $(0.72 \mathrm{mg} / \mathrm{g})$ and trace amount of IP4 $(0.09 \mathrm{mg} / \mathrm{g}$ ) (Ayet et al., 1997).

\subsection{Phytosterols}

Phytosterols, primarily $\beta$-sitosterol, campesterol, and stigmasterol, are integral natural components of plant cell membranes. They are proposed to have a wide range of biological effects including anti-inflammatory, anti-oxidative, anti-carcinogenic activities 
and cholesterol-lowering ability (Berger et al., 2004). Studies have shown that phytosterols can inhibit the intestinal absorption of cholesterol, thus lowering total plasma cholesterol and low-density lipoprotein (LDL) levels (de Jong et al., 2003). Pulses including lentils are one of the important dietary sources of phytosterols, along with vegetable oils, nuts and cereal grains. $\beta$-Sitosterol was found to be the most prevalent phytosterol in lentils, of which the concentration was $123.4 \mathrm{mg} / 100 \mathrm{~g}$, followed by $20.0 \mathrm{mg} / 100 \mathrm{~g}$ of stigmasterol and $15.0 \mathrm{mg} / 100 \mathrm{~g}$ of campesterol (Ryan et al., 2007). In another study, Kalogeropoulos et al. found that the predominant $\beta$-sitosterol ranged from 15.4 to $24.2 \mathrm{mg} / 100 \mathrm{~g}$ in cooked lentils, whereas the contents of stigmasterol and campesterol ranged from $2.60-2.63$ to $2.18-2.58 \mathrm{mg} / 100 \mathrm{~g}$, respectively (Kalogeropoulos et al., 2010).

\section{Processing effects on phytochemicals in lentils}

Lentils are usually processed before consumption. Processing not only improves the flavour and palatability of lentils, but also significantly affects their phytochemical content and profiles, as well as the antioxidant capacities. The stability of phytochemicals during processing has been a major concern. Han et al. reported that the total antioxidant activity and total phenolic content (TPC) of lentils was reduced by ca. $80,16-41$ and $22-42 \%$ by decortication, cooking and soaking, respectively (Han and Baik, 2008). Interestingly, they observed a loss of $94.8 \%$ in the total antioxidant activity of bound phenolics of lentils by decortication, indicating that most of the bound phytochemicals are distributed in the seed coat, whereas the total antioxidant activity of free phenolics increased by $10-36 \%$ in lentils after cooking probably due to the release of the conjugated phenolics during cooking. Xu et al. (2009) compared the influences of four thermal processing methods (conventional boiling, conventional steaming, pressure boiling, and pressure steaming) on phytochemical profiles, antioxidant capacities, and antiproliferation properties of commonly consumed cool-season food legumes including lentils. All thermal processing resulted in significant $(\mathrm{P}<0.05)$ reductions in total phenolic, procyanidin, total saponin, phytic acid, chemical antioxidant capacities (ferric reducing antioxidant power, FRAP; and peroxyl radical scavenging capacity, ORAC), and cellular antioxidant activity (CAA), as well as anti-proliferation capacities of lentils, as compared to raw lentils. Conventional boiling, pressure boiling and pressure steaming caused significant $(\mathrm{p}<0.05)$ decreases in gallic, chlorogenic, sinapic, $p$-coumaric acid, subtotal, and total phenolic acids, and significant increases in 2,3,4-trihydroxybenzoic acid, whereas conventional steaming did not cause significant changes in the chlorogenic and sinapic acid, subtotal cinnamic acid and total phenolic acid. Different thermal processing methods present significant differences. Their results indicated that steaming was a better cooking method than boiling in retaining antioxidants and phenolic components, whereas boiling was effective in reducing saponin and phytic acid contents (Xu and Chang, 2009). Our recent study found that cooking favours the release of carotenoids, tocopherols and flavonols (kaempferol glycosides) but leads to losses of flavanols (monomeric and condensed tannin) (Zhang et al., 2014b). However, a significantly reduction in tocopherols including $\alpha-, \beta-, \gamma$ - and $\delta$-tocopherols resulted from cooking was observed by FernandezOrozco et al. (2003). Elhardallou and Walker (1994) determined a loss of $60.5 \%$ in phytic acid in lentils during autoclaved cooking. Ruiz et al. (1996) observed that soaking did not modify the saponin content or composition of lentils regardless of the $\mathrm{pH}$ of the soaking solution. The native soyasaponin VI in lentils, however, was found to be partially degraded into soyasaponin I during cooking, and an overall loss of $15-31 \%$ in total saponin content was found for lentils.

Exogenous enzyme treatment and germination could also have significant effects on the phytochemicals in lentils. Dueñas et al. investigated the effect of the enzymes tannase, $\alpha$-galactosidase, phytase and viscozyme on the phenolic composition of lentils, and found that all exogenous enzyme treatment changed phenolic composition of lentil flours, particularly those of the hydroxycinnamic compounds and proanthocyanidins that are significantly decreased after the enzymatic treatments, whereas quercetin 3-O-rutinoside and luteolin increased and reached the highest concentration by treatment of tannase. Trans-resveratrol was only observed in the lentils treated by the tannase and phytase, and gallic acid was formed by the action of phytase, $\alpha$-galactosidase and tannase. The treatments of viscozyme, $\alpha$-galactosidase or tannase increased the antioxidant capacity as compared to raw lentils, and the quercetin 3-O-rutinoside was evident to be the main compound affecting antioxidant activity (Dueñas et al., 2007). After addition of commercial phytase to lentil flour, a reduction of 85-91, 57-69 and $6-27 \%$ in IP6, IP5 and IP4, respectively, was observed, whilst did not significantly change the content of IP3 (Frias et al., 2003). In general, the phenolic content in lentils tend to steadily decline during germination due to leaching into the soaking water, binding with other organic substances such as carbohydrates or proteins, and the activation of endogenous enzymes such as hydroxylases and polyphenoloxydases. López-Amorós et al. (2006) reported a general decrease in all of the lentil phenolics including hydroxybenzoic acids, hydroxycinnamic acids, $(+)$-catechin and procyanidin oligomers after soaking when compared with raw seeds, thereafter germination resulted in an overall increase of phenolics in lentils, with the exception of protocatechuic acid where no increase took place. It is worth noting that some of hydroxycinnamic compounds such as transp-coumaric acid and trans-ferulic acid, which had decreased or disappeared after soaking, were detected and increased from the beginning of the germination period. The hydroxycinnamic compounds are the constituents of plant cell walls, in various bonds and esterified forms, linked to arabinoxylans and lignin. The changes observed in these hydroxycinnamic compounds during germination therefore could be well explained by the action of the endogenous esterases. Yeo et al. (2015) even proposed a new indicator, the ratio of insoluble bound phenolics (IBPs) to soluble phenolics (SPs), to monitor changes in the antioxidant activity of lentils during germination. They observed an increase of TPC in both SPs (from 3.35 to $4.25 \mathrm{mg} \mathrm{GAE} / \mathrm{g}$ of defatted weight (DW)) and IBPs (from 4.78 to $6.45 \mathrm{mg} \mathrm{GAE} / \mathrm{g}$ of DW) of lentils during germination. Total flavonoids contents (TFC) of SPs decreased from 2.49 to $1.96 \mathrm{mg} \mathrm{CE} / \mathrm{g}$ of DW during the 4 days of germination probably due to the degradation of flavonoids by oxidants such as ROS produced in the mitochondria, whereas that of IBPs increased from 2.98 to $3.85 \mathrm{CE} \mathrm{mg/g} \mathrm{(Yeo} \mathrm{and} \mathrm{Shahidi,}$ 2015). Cevallos-Casals and Cisneros-Zevallos (2010) found that TPC of SPs from green lentils was increased significantly during 7 days germination. The condition of germination and illumination was found to have varied effects on the phenolic components of lentils and their biological activity. Phenolics were stimulated by cultivation under continuous light, and the content of $p$-hydroxybenzoic, benzoic and caffeic acids was significant increased on days 3 and 4 after germination (Świeca et al., 2012). Additionally, the contents of $\beta$-, $\gamma$ - and $\delta$-tocopherols were reported to decrease by $33.6-42.5,29.3-55.4$ and $23.7-47.1 \%$, respectively, in germinated lentil seeds of all cultivars, but the contents of $\alpha$-tocopherol increased by $1.6-48.9 \%$ in germinated seeds when compared to that of the raw lentils of all cultivars being investigated (Fernan- 
dez-Orozco et al., 2003). Ayet et al. (1997) observed that germinated lentil seeds at day 6 contained highest levels of soyasapogenol $\mathrm{B}$, whereas total phytic acid amounts were greatly reduced after 6 days germination.

\section{Antioxidant effects}

Given the fact that in situ analysis of the antioxidant activity in vivo is currently impossible, the antioxidant properties of phytochemicals in plant was mostly evaluated by chemical-based assays, such as Trolox equivalent antioxidant capacity (TEAC), 2,2-diphenyl1-picrylhydrazyl radical scavenging (DPPH), FRAP, ORAC, total radical-trapping antioxidant parameter (TRAP), inhibition of photochemiluminescence (PCL), inhibition of oxidation of human low-density lipoprotein cholesterol (LDL) and DNA, and iron(II) chelation activity. These antioxidant assay methods are mainly based on two mechanisms, the hydrogen atom transfer (HAT) and the single electron transfer (SET). In the methods of ORAC and PCL, lentil phytochemicals may act as free radical scavengers by donating a hydrogen atom, while assays that determine the ability to inhibit LDL and DNA oxidation by lentil phytochemicals, the antioxidant properties may be based on both hydrogen donation and metal chelation. Commonly used methods such as TEAC and FRAP are considered to detect the ability of lentil phytochemicals to transfer single electron (SET) to reduce any compound including metals, carbonyls and free radicals, whereas DPPH method is considered to follow both HAT and SET system. Several secondary constituents in lentil, mainly phenolics, appeared to serve as powerful antioxidants by preventing against oxidative and free radical mediated reactions. Most of researches on the phenolic profiles of lentils hitherto overviewed use these chemical-based methods to determine the antioxidant activities. These methods may give different equivalent numbers. The total antioxidant activity determined by the ABTS (2,2'-azinobis-3-ethyl-benzthiazoline6 -sulfonic acid) assay was highest in lentils at around $14 \mu \mathrm{mol}$ Trolox equivalent antioxidant capacity (TEAC)/g among 5 tested legumes, and insoluble-bound phenolics contributed $82-85 \%$ of total antioxidant activity in lentils (Han and Baik, 2008). These findings were also confirmed by Pellegrini et al. who observed that lentils had the highest total antioxidant capacity measured by FRAP and TRAP among tested pulses, but came second to broad beans by TEAC. Similarly, Xu et al. reported that lentils had the highest DPPH and ORAC activity in comparison with green pea, yellow pea and chickpea (Xu and Chang, 2008). Alshikh et al. (2015) found that all fractions including free, esterified and insoluble-bound from selected lentils showed varied reducing power and scavenging activity against DPPH, hydroxyl radicals and ABTS radical cation. Their potential bioactivity was further confirmed through inhibition of cupric ion induced human LDL peroxidation and peroxyl radical induced DNA strand breakage. According to our recent studies, the DPPH, FRAP and ORAC values of phenolics in lentils were in the range of 23.83-35.03 $\mu \mathrm{mol} \mathrm{TE} / \mathrm{g}$ DW, 18.75-34.52 AAE/g DW and 105.06-168.03 $\mu \mathrm{mol} \mathrm{TE/g} \mathrm{DW,}$ respectively, whilst the antioxidant activities of lentil hydrophobic fraction containing carotenoids and tocopherols were determined as 3.61-4.48 $\mu \mathrm{mol} \mathrm{TE} / \mathrm{g}$ DW and 2.73-6.23 $\mu \mathrm{mol} \mathrm{TE} / \mathrm{g}$ DW in the DPPH and PCL assay, respectively (Zhang et al., 2014a; Zhang et al., 2015).

CAA has recently been developed for the evaluation of antioxidant activity to overcome the lack of biological and physiological relevance of the chemical-based assays,. This method measures the ability of antioxidants to prevent the formation of fluorescent dichlorofluorescein (DCF) by 2, 20-azobis (2-amidinopropane) dihydrochloride (ABAP)-generated peroxyl radicals in live cell lines. CAA is considered more biologically relevant than the chemistrybased assays due to accounting of the absorption, metabolism and location of antioxidants inside a living cell. Lentil was reported to exhibit the greatest CAA with the lowest $\mathrm{IC}_{50}$ value $(0.67 \mathrm{mg} / \mathrm{mL})$, followed by yellow pea and green pea, whereas raw chickpea did not exhibit dose-dependent CAA. Pressurized steaming reduced CAA of lentil with increased $\mathrm{IC}_{50}$ value of $1.88 \mathrm{mg} / \mathrm{mL}$ (Xu and Chang, 2009). The same authors determined CAA of 11 selected lentil cultivars with the $\mathrm{IC}_{50}$ values ranging from 0.30 to $1.41 \mathrm{mg} /$ $\mathrm{mL}$, and revealed that the CAA results significantly correlated with chemical antioxidant assay ORAC (Xu and Chang, 2010).

\section{Anti-inflammatory effects}

Inflammation is known as a basic defense mechanism of responses to infection, burn, toxic chemicals, allergens or other noxious stimuli. However, excessive or persistent inflammation may lead to tissue destruction and many chronic diseases. There are two scenarios when the inflammatory response itself damages host tissue and causes organ dysfunction. One being an extremely acute or subacute inflammatory response that occurs when there is severe attacks from pathogens (sepsis) or debris from damaged host cells, and the other is pathologic inflammation or low grade inflammation, which when regulation of its pathways is disrupted, triggers chronic diseases such as atherosclerosis, type 2 diabetes and Alzheimer's disease (Tabas and Glass, 2013). As inflammatory reactions often include the formation of tissue-damaging oxidation products, i.e. increased oxidative stress, compounds with high antioxidant activity may inhibit inflammation. Interestingly, lentils have long been used by ancient treatment remedies to treat some inflammatory symptoms, such as skin infections by its water paste and the treatment of burns, after being roasted, milled and applied directly to affected areas (Sezik et al., 2001; Teklehaymanot et al., 2007). Additionally, regular consumption of pulse foods, particularly lentils, have been evidenced by many researches to reduce the incidence of developing chronic inflammatory disease including type 2 diabetes, cardiovascular diseases (CVD) and cancers (Adebamowo et al., 2005; Anderson et al., 2007; Rizkalla et al., 2003). Phenolic-rich lentils have the potential to reduce blood pressure due to its angiotensin I-converting enzyme (ACE) inhibitor activity (Boye et al., 2010; Hanson et al., 2014). The recent study observed that bioactive compounds such as legumin, vicilin and convicilin in lentil present higher ACE-inhibitory and cardioprotective activity (Garcia-Mora et al., 2014). In a hypertensive animal model, lentils administration can reduced the total cholesterol (TC), triglycerides (TG), low density lipoprotein (LDL) and pathological manifestations of cardio-morphometric analysis (Lukito et al., 2001). The specific bioactive components that exert these protective benefits on inflammatory-related diseases still need to be further identified and the mechanisms explored. Cyclooxygenases (COX) and lipoxygenase (LOX) are two major metabolic routes controlling eicosanoid biosynthesis. COX-1 and COX-2 regulate inflammatory responses differentially, thus different types of inhibitors are required for the anti-inflammatory effects. COX2 inhibitors increase inflammation risk whereas COX-1 inhibitors reduce it. COX-2 is a key enzyme catalyzing the production of prostaglandins $(\mathrm{PG})$ in response to inflammatory stimuli (Surh, 2002). The inhibition of prostaglandin E2 (PGE2) and nitric oxide $(\mathrm{NO})$ production has been considered a potential therapy for different inflammatory disorders. While the nuclear transcription 
factor $(\mathrm{NF}-\mathrm{\kappa B})$ is the target of the intracellular signaling pathways responsible for induction of COX-2 expression, it can be a positive regulator of COX-2 in diverse cell types (Surh et al., 2001). Many studies reported that plant-derived phenolics and flavonoids exhibit excellent anti-inflammatory activity by regulating the levels of various inflammatory cytokines or mediators including IL-1, IL-6, IL-10, TNF- $\alpha$, NF- $\mathrm{B}$, NO, iNOS, LOX, COX-1 and COX-2 (Wu et al., 2011). Boudjou et al. (2013) found that aqueous ethanol $(80 \%)$ extract of lentil hulls exhibited high anti-inflammatory activities preferentially inhibiting $15-\mathrm{LOX}\left(\mathrm{IC}_{50}, 55 \mu \mathrm{g} / \mathrm{ml}\right)$, with moderate COX-1 $\left(\mathrm{IC}_{50}, 66 \mu \mathrm{g} / \mathrm{ml}\right)$ and weak COX-2 $\left(\mathrm{IC}_{50}, 119 \mu \mathrm{g} /\right.$ $\mathrm{ml}$ ) inhibitory effects on the COX pathway. Current research on the anti-inflammatory activity of lentil is limited to the in vitro studies of phenolics fraction. We have recently reported that phenolics of lentils showed dose-dependent anti-inflammatory activity against pro-inflammatory cytokines COX-2, IL- $1 \beta$ and IL- 6 in TNF- $\alpha-$ induced inflammation in Caco- 2 cells. The antioxidant and anti-inflammatory activities were positively correlated with the total and individual phenolic contents (Zhang et al. 2017). Concisely, these studies suggest that the dietary consumption of polyphenolic-rich lentils should be on a regular basis, having the potential to reduce the risk of inflammatory-related chronic diseases. More in vitro and in vivo studies are needed to investigate the anti-inflammatory mechanisms of different phytochemicals in lentils.

\section{Interaction with microbiome}

The human intestinal tract is home to more than 100 trillion microorganisms. Gut microbes are believed to be involved in major physiological activities, such as protecting gut epithelial cells from pathogens invasion, stimulating the immune system, increasing nutrient availability, stimulating bowel motility. After consumption, foods containing phenolics may undergo a serial of enzyme reactions, following alteration of physiochemical properties in the digestive tract, including the mouth, stomach, small intestinal and large intestine (colon). Those free Phenolic compounds are released from the food matrix in the stomach and small intestinal (gastrointestinal tract) by enzymes and acidic or base conditions, whereas majority of insoluble-bound phenolics survive in gastrointestinal tract digestion and transfer into the colon (large intestine), and release single phenolics or metabolits by the activity of digestive enzymes or colon microbiota. It was reported that only $5-10 \%$ of free phenolics can be absorbed in the small intestine, while the remaining $90 \%-95 \%$ degrade and move directly to the colon (Scalbert, A. \& Williamson, G. 2000). Therefore, the interaction between the insoluble-bound phenolics and microbiome might play a critical role in the potential health benefits of lentils. The metabolism and absorption mechanism of insoluble-bound phenolics of lentils in colon after microbiota fermentation has not yet been well studied, which is worth investigating in future research.

\section{Summary}

The above review on the phytochemical composition, as well as their antioxidant and anti-inflammatory activity, indeed showed that lentils contain a plethora of health promoting bioactives in addition to the macronutrients such as starch and protein. Many factors can affect the health benefits of lentils, as the content and composition of phenolics, carotenoids, tocopherols and other nutrients hitherto mentioned can vary significantly in different cultivars, and by different processing methods. Lentils have been traditionally consumed as whole seeds, however, processing of lentil into different fractions, i.e. flour, protein and starch, has been in steady sharp growth in recent years due to increased consumer needs in alternative plant protein (other than soy protein) and specialty foods such as gluten-free foods. Lentil processing generates significant amount of seed coats which are currently of no or low value, yet studies have shown that these are the major source of dietary fiber and antioxidant and anti-inflammatory phenolics, in both free and insoluble-bound forms. The high content of bound phenolics, and the in vitro antioxidant and anti-inflammatory effects of lentil hulls warrant further studies in vivo. In addition, potential synergistic effects may exist among different classes of phytochemicals. Researches on the potential health benefits of lentil bioactives have not paid much attention to the bioaccessibility, bioavailability in vivo. In order to be absorbed and to reach the target cells or tissues at a certain level for these compounds, they have to survive the gastrointestinal tract, and be uptaken and transported. This requires a multidisciplinary in approach in future studies.

\section{Acknowledgments}

We thank Pulse Canada for providing the lentil samples. This project is supported by the A-Base Project (\#J-001322.001.04) of Agriculture \& Agri-Food Canada.

\section{References}

Adebamowo, C.A., Cho, E., Sampson, L., Katan, M.B., Spiegelman, D., Willett, W.C., and Holmes, M.D. (2005). Dietary flavonols and flavonolrich foods intake and the risk of breast cancer. Int. J. Cancer 114(4): 628-633.

Aguilera, Y., Dueñas, M., Estrella, I., Hernández, T., Benitez, V., Esteban, R.M., and Martín-Cabrejas, M.A. (2010). Evaluation of phenolic profile and antioxidant properties of Pardina lentil as affected by industrial dehydration. J. Agric. Food Chem. 58(18): 10101-10108.

Alshikh, N., de Camargo, A.C., and Shahidi, F. (2015). Phenolics of selected lentil cultivars: Antioxidant activities and inhibition of low-density lipoprotein and DNA damage. J. Funct. Foods 18: 1022-1038.

Amarowicz, R., Estrella, I., Hernández, T., Dueñas, M., Troszyńska, A. Kosińska, A., and Pegg, R.B. (2009). Antioxidant activity of a red lentil extract and its fractions. Int. J. Mol. Sci. 10(12): 5513-5527.

Amarowicz, R., Estrella, I., Hernández, T., Robredo, S., Troszyńska, A. Kosińska, A., and Pegg, R.B. (2010). Free radical-scavenging capacity, antioxidant activity, and phenolic composition of green lentil (Lens culinaris). Food Chem. 121(3): 705-711.

Amarowicz, R., and Pegg, R.B. (2008). Legumes as a source of natural antioxidants. Eur. J. Lipid Sci. Technol. 110(10): 865-878.

Amarowicz, R., Troszynska, A., Barylko-Pikienla, N., and Shahidi, F. (2004). Polyphenolics extracts from legume seeds: correlations between total antioxidant activity, total phenolics content, tannins content and astringency. J. Food Lipids 11(4): 278-286.

Anderson, J.W., and Major, W.A. (2007). Pulses and lipaemia, short- and long-term effect: Potential in the prevention of cardiovascular disease. Br. J. Nutr. S3: 263-271.

Andreasen, M.F., Kroon, P.A., Williamson, G., and Garcia-Conesa, M.-T. (2001). Esterase activity able to hydrolyze dietary antioxidant hydroxycinnamates is distributed along the intestine of mammals. J. Agric. Food Chem. 49(11): 5679-5684.

Andreasen, M.F., Landbo, A.-K., Christensen, L.P., Hansen, Å., and Meyer A.S. (2001). Antioxidant effects of phenolic rye (Secale cereale L.) extracts, monomeric hydroxycinnamates, and ferulic acid dehydrodimers on human low-density lipoproteins. J. Agric. Food Chem. 49(8): 4090-4096.

Ayet, G., Burbano, C., Cuadrado, C., Pedrosa, M., Robredo, L., Muzquiz, M., De la Cuadra, C., Castano, A., and Osagie, A. (1997). Effect of ger- 
mination, under different environmental conditions, on saponins, phytic acid and tannins in lentils (Lens culinaris). J. Sci. Food Agric. 74(2): 273-279.

Bartolome, B., and Gómez-Cordovés, C. (1999). Barley spent grain: release of hydroxycinnamic acids (ferulic and p-coumaric acids) by commercial enzyme preparations. J. Sci. Food Agric. 79(3): 435-439.

Berger, A., Jones, P.J., and Abumweis, S.S. (2004). Plant sterols: factors affecting their efficacy and safety as functional food ingredients. Lipids Health Disease 3: 5.

Bhatty, R. (1988). Composition and quality of lentil (Lens culinaris Medik): a review. Can. Inst. Food Sci. Technol. J. 21(2): 144-160.

Biehler, E., Alkerwi, A., Hoffmann, L., Krause, E., Guillaume, M., Lair, M.L., and Bohn, T. (2012). Contribution of violaxanthin, neoxanthin, phytoene and phytofluene to total carotenoid intake: assessment in Luxembourg. J. Food Comp. Anal. 25(1): 56-65.

Boudjou, S., Oomah, B.D., Zaidi, F., and Hosseinian, F. (2013). Phenolics content and antioxidant and anti-inflammatory activities of legume fractions. Food Chem. 138(2-3): 1543-1550.

Boye, J.I., Roufik, S., Pesta, N., and Barbana, C. (2010). Angiotensin I-converting enzyme inhibitory properties andSDS-PAGE of red lentil protein hydrolysates. LWT-Food Sci. Technool. 43: 987-991.

Cevallos-Casals, B.A., and Cisneros-Zevallos, L. (2010). Impact of germination on phenolic content and antioxidant activity of 13 edible seed species. Food Chem. 119(4): 1485-1490.

D’Arcy, A., and Jay, M. (1978). Les flavonoïdes des graines de Lens culinaris. Phytochemistry 17(4): 826-827.

Dahl, W.J., Foster, L.M., and Tyler, R.T. (2012). Review of the health benefits of peas (Pisum sativum L.). Br. J. Nut. 108(S1): S3-S10.

de Jong, A., Plat, J., and Mensink, R.P. (2003). Metabolic effects of plant sterols and stanols (Review). J. Nutr. Biochem. 14(7): 362-369.

DellaPenna, D., and Pogson, B.J. (2006). Vitamin synthesis in plants: tocopherols and carotenoids. Annu. Rev. Plant Biol. 57: 711-738.

Dueñas, M., Hernández, T., and Estrella, I. (2002). Phenolic composition of the cotyledon and the seed coat of lentils (Lens culinaris L.). Eur. Food Res. Technol. 215(6): 478-483.

Dueñas, M., Hernández, T., and Estrella, I. (2007). Changes in the content of bioactive polyphenolic compounds of lentils by the action of exogenous enzymes. Effect on their antioxidant activity. Food Chem. 101(1): 90-97.

Dueñas, M., Sun, B., Hernández, T., Estrella, I., and Spranger, M.I. (2003). Proanthocyanidin composition in the seed coat of lentils (Lens culinaris L.). J. Agric. Food Chem. 51(27): 7999-8004.

Duenas, M., Hernandez, T., and Estrella, I. (2006). Assessment of in vitro antioxidant capacity of the seed coat and the cotyledon of legumes in relation to their phenolic contents. Food Chem. 98(1): 95-103.

Elhardallou, S.B., and Walker, A.F. (1994). Phytic acid content of three legumes in the raw, cooked and fibre forms. Phytochem. Anal. 5(5): 243-246.

Fernandez-Orozco, R., Zieliński, H., and Piskuła, M.K. (2003). Contribution of low-molecular-weight antioxidants to the antioxidant capacity of raw and processed lentil seeds. Food/Nahrung 47(5): 291-299.

Frias, J., Doblado, R., Antezana, J.R., and Vidal-Valverde, C. (2003). Inositol phosphate degradation by the action of phytase enzyme in legume seeds. Food Chem. 81(2): 233-239.

Garcia-Mora, P., Penas, E., Frias, J., and Martinez-Villaluenga, C. (2014). Savinase, the most suitable enzyme for releasing peptides from lentil (Lens culinaris var. Castellana) protein concentrates with multifunctional properties. J. Agric. Food Chem. 62: 4166-4174.

Graf, E., and Eaton, J.W. (1990). Antioxidant functions of phytic acid. Free Rad. Biol. Med. 8(1): 61-69.

Gumienna, M., Lasik, M., and Czarnecki, Z. (2009). Influence of plant extracts addition on the antioxidative properties of products obtained from green lentil seeds during in vitro digestion process. Pol. J.Food Nutr. Sci. 59(4): 295-298.

Han, H., and Baik, B.K. (2008). Antioxidant activity and phenolic content of lentils (Lens culinaris), chickpeas (Cicer arietinum L.), peas (Pisum sativum L.) and soybeans (Glycine max), and their quantitative changes during processing. Int. J. Food Sci. Technol. 43(11): 1971-1978.

Hanson, M.G., Zahradka, P., and Taylor, C.G. (2014). Lentil-based diets attenuate hypertension and large-artery remodelling in spontaneously hypertensive rats. Br. J. Nutr. 111: 690-698.
Kalogeropoulos, N., Chiou, A., Ioannou, M., Karathanos, V.T., Hassapidou, M., and Andrikopoulos, N.K. (2010). Nutritional evaluation and bioactive microconstituents (phytosterols, tocopherols, polyphenols, triterpenic acids) in cooked dry legumes usually consumed in the Mediterranean countries. Food Chem. 121(3): 682-690.

López-Amorós, M., Hernández, T., and Estrella, I. (2006). Effect of germination on legume phenolic compounds and their antioxidant activity. J. Food Comp. Anal. 19(4): 277-283.

Lukito, W. (2001). Candidate foods in the Asia-Pacific region for cardiovascular protection: Nuts, soy, lentils, and tempe. Asia Pacific J. Clin. Nutr. 10: 128-133.

Marathe, S.A., Rajalakshmi, V., Jamdar, S.N., and Sharma, A. (2011). Comparative study on antioxidant activity of different varieties of commonly consumed legumes in India. Food Chem. Toxicol. 49(9): 2005-2012.

Mirali, M., Ambrose, S.J., Wood, S.A., Vandenberg, A., and Purves, R.W. (2014). Development of a fast extraction method and optimization of liquid chromatography-mass spectrometry for the analysis of phenolic compounds in lentil seed coats. J. Chromatogr. B 969: 149-161.

Mudryj, A.N., Yu, N., Hartman, T.J., Mitchell, D.C., Lawrence, F.R., and Aukema, H.M. (2012). Pulse consumption in Canadian adults influences nutrient intakes. Br. J. Nutr. 108(S1): S27-S36.

Rizkalla, S.W., Bellisle, F., and Slama, G. (2003). Health benefits of low glycaemic index foods, such as pulse in diabetic patients and healthy individuals. Br. J. Nutr. 88(S3): 255-262.

Rochfort, S., and Panozzo, J. (2007). Phytochemicals for health, the role of pulses. J. Agric. Food Chem. 55(20): 7981-7994.

Ruiz, R.G., Price, K.R., Arthur, A.E., Rose, M.E., Rhodes, M.J., and Fenwick, R.G. (1996). Effect of soaking and cooking on the saponin content and composition of chickpeas (Cicer arietinum) and lentils (Lens culinaris). J. Agric. Food Chem. 44(6): 1526-1530.

Ruiz, R.G., Price, K.R., Rose, M.E., and Fenwick, G.R. (1997). Effect of seed size and testa colour on saponin content of Spanish lentil seed. Food Chem. 58(3): 223-226.

Ryan, E., Galvin, K., O'Connor, T., Maguire, A., and O'Brien, N. (2007). Phytosterol, squalene, tocopherol content and fatty acid profile of selected seeds, grains, and legumes. Plant Foods Hum. Nutr. 62(3): 85-91.

Sagratini, G., Zuo, Y., Caprioli, G., Cristalli, G., Giardinà, D., Maggi, F., Molin, L., Ricciutelli, M., Traldi, P., and Vittori, S. (2009). Quantification of Soyasaponins I and $\beta g$ in Italian Lentil Seeds by Solid-Phase Extraction (SPE) and High-Performance Liquid Chromatography- Mass Spectrometry (HPLC-MS). J. Agric. Food Chem., 57( 23): 11226-11233.

Scalbert, A., and Williamson, G. (2000). Dietary Intake and Bioavailability of Polyphenols. J. Nutr. 130(8): 2073S-2085S.

Schneider, A.V. (2002). Overview of the market and consumption of puises in Europe. Br. J. Nutr. 88(S3): 243-250.

Sezik, E., Yeşilada, E., Honda, G., Takaishi, Y., Takeda, Y., and Tanaka, T. (2001). Traditional medicine in Turkey X. Folk medicine in Central Anatolia. J. Ethnopharmacol. 75(2-3): 95-115.

Surh, Y.-J. (2002). Anti-tumor promoting potential of selected spice ingredients with antioxidative and anti-inflammatory activities: a short review. Food Chem. Toxicol. 40(8): 1091-1097.

Surh, Y.-J., Chun, K.-S., Cha, H.-H., Han, S.S., Keum, Y.-S., Park, K.-K., and Lee, S.S. (2001). Molecular mechanisms underlying chemopreventive activities of anti-inflammatory phytochemicals: down-regulation of COX-2 and iNOS through suppression of NF-kB activation. Mut. Res./ Fund. Mol. Mech. Mutagen. 480-481: 243-268.

Świeca, M., Gawlik-Dziki, U., Kowalczyk, D., and Złotek, U. (2012). Impact of germination time and type of illumination on the antioxidant compounds and antioxidant capacity of Lens culinaris sprouts. Sci. Horticul. 140: 87-95.

Tabas, I., and Glass, C.K. (2013). Anti-Inflammatory Therapy in Chronic Disease: Challenges and Opportunities. Science 339(6116): 166-172

Takeoka, G.R., Dao, L.T., Tamura, H., and Harden, L.A. (2005). Delphinidin 3-O-(2-O- $\beta$-D-glucopyranosyl- $\alpha$-L-arabinopyranoside): A novel anthocyanin identified in beluga black lentils. J. Agric. Food Chem. 53(12): 4932-4937

Teklehaymanot, T., Giday, M., Medhin, G., and Mekonnen, Y. (2007) Knowledge and use of medicinal plants by people around Debre Libanos monastery in Ethiopia. J. Ethnopharmacol. 111(2): 271-283. 
Thavarajah, D., Thavarajah, P., Sarker, A., and Vandenberg, A. (2009). Lentils (Lens culinaris Medikus Subspecies culinaris): a whole food for increased iron and zinc intake. J. Agric. Food Chem. 57(12): 5413-5419.

Villegas, R., Gao, Y.-T., Yang, G., Li, H.-L., Elasy, T.A., Zheng, W., and Shu, X.O. (2008). Legume and soy food intake and the incidence of type 2 diabetes in the Shanghai Women's Health Study. Amer. J. Clin. Nutri. 87(1): 162-167.

Wu, J., Li, J., Cai, Y., Pan, Y., Ye, F., Zhang, Y., Zhao, Y., Yang, S., Li, X., and Liang, G. (2011). Evaluation and Discovery of Novel Synthetic Chalcone Derivatives as Anti-Inflammatory Agents. J. Med. Chem. 54(23) 8110-8123.

Xu, B., and Chang, S. (2007). A comparative study on phenolic profiles and antioxidant activities of legumes as affected by extraction solvents. J. Food Sci. 72(2): S159-S166.

Xu, B., and Chang, S.K. (2009). Phytochemical profiles and health-promoting effects of cool-season food legumes as influenced by thermal processing. J. Agric. Food Chem. 57(22): 10718-10731.

$\mathrm{Xu}, \mathrm{B}$., and Chang, S.K. (2010). Phenolic substance characterization and chemical and cell-based antioxidant activities of 11 lentils grown in the Northern United States. J. Agric. Food Chem. 58(3): 1509-1517.

$\mathrm{Xu}$, B., and Chang, S.K. (2012). Comparative study on antiproliferation properties and cellular antioxidant activities of commonly consumed food legumes against nine human cancer cell lines. Food Chem. 134(3): 1287-1296.

Xu, B., and Chang, S.K.C. (2008). Effect of soaking, boiling, and steaming on total phenolic contentand antioxidant activities of cool season food legumes. Food Chem. 110(1): 1-13.
Yeo, J., and Shahidi, F. (2015). Critical Evaluation of Changes in the Ratio of Insoluble Bound to Soluble Phenolics on Antioxidant Activity of Lentils during Germination. J. Agric. Food Chem. 63(2): 379-381.

Zhang, B., Deng, Z., Ramdath, D.D., Tang, Y., Chen, P.X., Liu, R., Liu, Q., and Tsao, R. (2015). Phenolic profiles of 20 Canadian lentil cultivars and their contribution to antioxidant activity and inhibitory effects on $\alpha$-glucosidase and pancreatic lipase. Food Chem. 172: 862-872.

Zhang, B., Deng, Z., Tang, Y., Chen, P., Liu, R., Ramdath, D.D., Liu, Q., Hernandez, M., and Tsao, R. (2014a). Fatty acid, carotenoid and tocopherol compositions of 20 Canadian lentil cultivars and synergistic contribution to antioxidant activities. Food Chem. 161: 296-304.

Zhang, B., Deng, Z., Tang, Y., Chen, P.X., Liu, R., Ramdath, D.D., Liu, Q., Hernandez, M., and Tsao, R. (2014b). Effect of domestic cooking on carotenoids, tocopherols, fatty acids, phenolics, and antioxidant activities of lentils (Lens culinaris). J. Agric. Food Chem. 62(52): 1258512594.

Zhang, B., Deng, Z., Tang, Y., Chen, P.X., Ramdath, D.D., Liu, R., Liu, Q., Hernandez, M., and Tsao, R. (2017). Bioaccessibility, in vitro antioxidant and anti-inflammatory activities of phenolics in cooked green lentil (Lens culinaris). J. Funct. Foods 32: 248-255.

Zou, Y., Chang, S.K., Gu, Y., and Qian, S.Y. (2011). Antioxidant activity and phenolic compositions of lentil (Lens culinaris var. Morton) extract and its fractions. J. Agric. Food Chem. 59(6): 2268-2276.

Zupfer, J., Churchill, K., Rasmusson, D., and Fulcher, R. (1998). Variation in ferulic acid concentration among diverse barley cultivars measured by HPLC and microspectrophotometry. J. Agric. Food Chem. 46(4): 1350-1354. 